\title{
Invertebrate 7SK snRNAs
}

\author{
Andreas R. Gruber - Dorota Koper-Emde - Manja Marz · Hakim Tafer · \\ Stephan Bernhart · Gregor Obernosterer · Axel Mosig · Ivo L. Hofacker · \\ Peter F. Stadler · Bernd-Joachim Benecke
}

Received: 24 July 2007 / Accepted: 5 October 2007/Published online: 12 January 2008

(C) Springer Science+Business Media, LLC 2008

\begin{abstract}
SK RNA is a highly abundant noncoding RNA in mammalian cells whose function in transcriptional regulation has only recently been elucidated. Despite its highly conserved sequence throughout vertebrates, all attempts to discover 7SK RNA homologues in invertebrate species have failed so far. Here we report on a combined experimental and computational survey that succeeded in discovering 7SK RNAs in most of the major deuterostome clades and in two protostome phyla: mollusks and annelids. Despite major efforts, no candidates were found in any of the many available ecdysozoan genomes, however. The additional sequence data confirm the evolutionary conservation and hence functional importance of the previously described $3^{\prime}$ and $5^{\prime}$ stemloop motifs, and provide evidence for a third, structurally well-conserved domain.
\end{abstract}

Andreas R. Gruber, Dorota Koper-Emde, Manja Marz, and Hakim Tafer contributed equally.

A. R. Gruber $(\bowtie) \cdot$ H. Tafer $\cdot$ S. Bernhart .

I. L. Hofacker ? P. F. Stadler

Institute for Theoretical Chemistry, University of Vienna,

Währingerstrasse 17, A-1090 Wien, Austria

e-mail: agruber@tbi.univie.ac.at

D. Koper-Emde · B.-J. Benecke

Biochemistry NC6/132, Ruhr-University Bochum, Bochum, Germany

M. Marz · S. Bernhart · P. F. Stadler

Bioinformatics Group, Department of Computer Science, and Interdisciplinary Center of Bioinformatics, University of Leipzig, Härtelstrasse 16-18, D-04107 Leipzig, Germany

G. Obernosterer

Institute of Molecular Biotechnology of the Austrian Academy

of Sciences (IMBA), A-1030 Vienna, Austria
Keywords 7SK RNA · Polymerase III transcription · Noncoding RNA · Lophotrochozoans

\section{Introduction}

The 7SK snRNA is a highly abundant noncoding RNA in vertebrate cells. The polymerase (Pol) III transcript, with a length of about 330 nucleotides (nt) (Krüger and Benecke 1987; Murphy et al. 1987), is highly conserved in vertebrates (Gürsoy et al. 2000). Due to its abundance, it has been known since the 1960s. Its function as a transcriptional regulator, however, has only recently been discovered. 7SK mediates the inhibition of the general transcription elongation factor P-TEFb by the HEXIM1 protein and thereby represses transcript elongation by Pol
A. Mosig

Department of Combinatorics and Geometry (DCG), MPG/CAS Partner Institute for Computational Biology (PICB), Shanghai Institutes for Biological Sciences (SIBS) Campus, Shanghai, China

\section{A. Mosig}

Max Planck Insitute for Mathematics in the Sciences,

Inselstrasse 22, D-04103 Leipzig, Germany

P. F. Stadler

RNomics Group, Fraunhofer Institut für Zelltherapie und Immunologie-IZI Deutscher Platz 5e, D-04103 Leipzig, Germany

P. F. Stadler

Santa Fe Institute, 1399 Hyde Park Road, Santa Fe, NM 87501, USA 
II (Blazek et al. 2005; Egloff et al. 2006; Michels et al. 2004; Peterlin and Price 2006). Furthermore, 7SK RNA suppresses the deaminase activity of APOBEC 3C and sequesters this enzyme in the nucleolus (He et al. 2006).

Two distinct secondary structure elements are highly conserved throughout vertebrates (Egloff et al. 2006): a 5'terminal hairpin structure that binds both HEXIM1 and P$\mathrm{TEFb}$, and a $3^{\prime}$-terminal hairpin that interacts with $\mathrm{P}-\mathrm{TEFb}$ only. In contrast to the nearly perfect sequence conservation in jawed vertebrates, the 7SK RNA from the lamprey Lampetra fluviatilis differed in more than $30 \%$ of its nucleotide positions from its mammalian counterpart (Gürsoy et al. 2000). The highest sequence conservation is observed in the $5^{\prime}$ and $3^{\prime}$ hairpin regions. The sequence conservation seems to decline rapidly outside the gnathostomes. In Gürsoy et al. (2000), some of us also reported on an unsuccessful attempt to find 7SK RNA in hagfish and lancet and suggested that the 7SK RNA might be a vertebrate innovation. In this paper we combine improved cloning strategies with systematic computational homology searches to detect highly divergent 7SK RNAs in invertebrate animals.

\section{Materials and Methods}

\section{Cloning and Sequencing of 7SK RNAs}

Total cellular RNA was isolated from frozen tissue, minced with scissors, and homogenized in buffer containing guanidinium thiocyanate (Chomczynski and Sacchi 1987). Northern blots were performed with $5 \mu \mathrm{g}$ of purified RNA separated in $2 \%$ agarose gels containing $0.67 \mathrm{M}$ formaldehyde. After transfer to nylon membranes (Hybond-N; Amersham), immobilized RNA was hybridized with labeled antisense RNA probes generated by T7 transcription of inversely cloned cDNA fragments of the previously identified 7SK RNA of Lampetra fluviatilis (Gürsoy et al. 2000). Hybridization with labeled antisense RNA $\left(2 \times 10^{6} \mathrm{cpm} / \mathrm{ml}\right)$ was in $50 \%$ formamide, $0.1 \% \operatorname{SDS}, 5 \times$ Denhardt's reagent, $10 \mu \mathrm{g} / \mathrm{ml}$ each of yeast tRNA and denatured salmon sperm DNA, and $5 \times$ SET $(150 \mathrm{mM}$ $\mathrm{NaCl}, 20 \mathrm{mM}$ Tris-HCl, pH 7.9, 1 mM EDTA).

Cloning of new 7SK cDNAs was based on RT-PCR reactions. In the first step $4 \mu \mathrm{g}$ of total RNA was reverse transcribed using the Omniscript RT-Kit (Qiagen) with a primer complementary to the $3^{\prime}$-end of 7SK RNA. An aliquot of that assay was taken for PCR with the same $3^{\prime}$ primer and a specific upstream primer. The successful combination for Myxine and Branchiostoma amplification was nt 54-72 as upstream primer and nt 197-215 as downstream primer, both of the human 7SK DNA sequence. For Helix, the same upstream primer, but in combination with a lamprey downstream primer (corresponding to positions 299-316), was successful. Candidate fragments were cloned, sequenced, and taken to deduce gene-specific "nested" primers for rapid amplification of cDNA ends (RACE) experiments (Frohman et al. 1988), as described earlier (Gürsoy et al. 2000). Briefly, for the $3^{\prime}$ ends cellular RNA was first polyadenylated and reverse transcription started with oligo(dT) carrying at its $5^{\prime}$-side an oligonucleotide sequence suitable for subsequent PCR with two "nested"' gene-specific primers. The $5^{\prime}$-ends were obtained by reverse transcription with a specific primer and oligonucleotide ligation (T4-RNA ligase) to the $3^{\prime}$-end of first-strand cDNAs. As before, PCR amplification was achieved with nested gene-specific primers.

\section{Computational Homology Search}

Homology search was performed as a stepwise procedure. In the first stage, we started with the sequence of the functional human 7SK sequence (X05490, X04236 (Krüger and Benecke 1987; Murphy et al. 1984; Wassarman and Steitz 1991; Zieve and Penman 1976)) and performed a blast search against the genome assemblies available in Ensembl (version 42). In this way, we identified candidates in other vertebrate genomes, including the following, previously published sequences: Mus musculus (M63671, Moon and Krause 1991), Rattus norvegicus (K02909, Reddy et al. 1984), Takifugu rubripes (AJ890104, Egloff et al. 2006; Myslinksi et al. 2004), Tetraodon nigroviridis (AJ890103, Egloff et al. 2006), Danio rerio (AJ890102, Egloff et al. 2006), and Gallus gallus (AJ890104, Egloff et al. 2006). In addition, we searched the shotgun traces of a selection of unfinished mammalian genomes as well as all unfinished nonmammalian animals. Beyond jawed vertebrates, this initial blast search recovered a single candidate in the genome of the lamprey Petromyzon marinus, which turned out the be very closely related to the published sequence of Lampetra fluviatilis 7SK RNA (Gürsoy et al. 2000). The match to a single shotgun trace (1047111637562) from the nematode Brugia malayi was disregarded since it exactly matched the human sequence and hence is certainly a contamination.

We then created a multiple sequence alignment starting with an initial CLUSTALW alignment. This was then manually refined to conform to the experimentally determined structure model of human 7SK snRNA by Wassarman and Steitz (1991). The best-conserved blocks were marked and converted in the search patterns using the aln2pattern program, which is part of the fragrep2 package (Mosig et al. 2007). This step was guided by the functional interpretation of the structural domains of the 7SK in Egloff et al. (2006). The program fragrep, which 

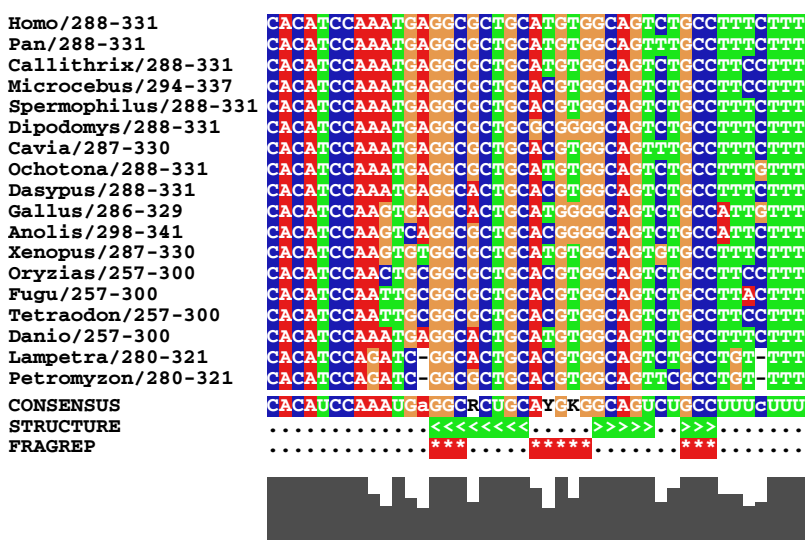

Fig. 1 Alignment and consensus sequence and structure of the $3^{\prime}$ hairpin of vertebrate 7SK RNAs

searches fragmented approximate sequence patterns in genomic DNA sequences, was then used to scan the available genomic DNA data.

First efforts focused on the 3 '-terminal hairpin structure (Fig. 1). Both the loop motive and the GGC-GCC stem have been shown to be crucial for P-TEFb binding and have little or no sequence variation. Search patterns for fragrep are specified as partial sequence patterns (using IUPAC notation), annotated by the minimal and maximal number of unspecified nucleotides separating the sequence motifs and the maximal number of mismatches and in/dels that are acceptable in each of the sequence motifs. The initial search pattern for the $3^{\prime}$ hairpin is indicated in the last line in Fig. 1.

The candidate sequences found by fragrep in Ciona intestinalis and Ciona savignyi were filtered manually by comparing them with the secondary structure model of the human 3'-terminal hairpin. The best matches also showed high sequence conservation in the part of the $5^{\prime}$-terminal hairpin structure responsible for HEXIM1 and P-TEFb binding (Egloff et al. 2006) and were clearly identifiable as homologues by aligning them with each other. We then extended our pattern to search for highly conserved regions in both the $5^{\prime}$ and the $3^{\prime}$ hairpin structures. The pattern was refined in an iterative way every time a new candidate sequences was found. The final search pattern is given in Fig. 2.

The blast and fragrep searches were performed on the genomic sequences from Ensembl and pre-Ensembl, (versions 44 and 45), the genomes of Branchiostoma floridae and Nematostella vectensis (downloaded from the Joint Genome Institute; ${ }^{1}$ ), and the metazoan sequences contained in the Ensembl trace archive. More details are given in the electronic supplement.

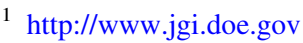

\begin{tabular}{|rrlll|}
\hline 6 & & & & \\
0 & 0 & GAUCGC & 0 & 0 \\
4 & 16 & GGC & 0 & 0 \\
1 & 2 & GAUCUG & 0 & 0 \\
150 & 250 & UCCA & 0 & 0 \\
0 & 5 & GGC & 0 & 0 \\
0 & 19 & GCC & 0 & 0 \\
\hline
\end{tabular}

Fig. 2 Final fragrep search pattern for 7SK RNAs, specifying six sequence motifs, their minimal and maximal separation, and the number of acceptable substitutions and in/dels. Here exact matches of the short motifs are required

\section{Computational Identification of Putative 7SK} Promoters

It is well known that the promoters of RNA pol III transcribed 7SK, U6, and U6atac snRNA contain three common elements: the proximal sequence element (PSE), about $50 \mathrm{nt}$ upstream of the gene; a TATA box-like element; and distal enhancer elements (Dahlberg and Lund 1988; Wassarman and Steitz 1991). The PSE of the Pol III snRNAs is very similar to that on the snRNA transcribed by Pol II (U1, U2, U4, U5, U11, U12, U4atac).

In order to distinguish functional 7SK genes from pseudogenes we investigated their upstream regions for snRNA-specific promoter elements. Since these sequence motifs can vary significantly (Hernandez Jr et al. 2007) between species, we searched the genomes also for spliceosomal snRNAs, extracted 100-nt upstream regions, and used meme (version 3.5.4) (Bailey and Elkan 1994) to identify the PSE consensus separately for each species. For the study reported here we used the applet available from http://meme.nbcr.net/downloads/ with default options and parameters -nmotifs 5 -minw 10 -maxw 30 . The PSE patterns obtained in this way were then used to identify those 7SK candidates that have an snRNA-like PSE. The results of the homology search for the spliceosomal RNA genes will be reported elsewhere (Marz et al. 2007).

\section{Structural Alignments}

A structural alignment of the vertebrate sequences based on the experimentally determined structure for human 7SK snRNA (Krüger and Benecke 1987; Wassarman and Steitz 1991) was constructed manually using the RALEE mode (Griffiths-Jones 2005) for the emacs editor. The model was iteratively improved upon the addition of new candidate sequences during analysis with the help of consensus structure predictions using RNAalifold (Hofacker et al. 2002).

The $5^{\prime}$ stem sequence of the basal deuterostomes and lophotrochozoa diverged too much from the vertebrate consensus, so they could not be aligned based on sequence 
similarity alone. We therefore used the absolutely conserved GATC-GATC stem in the center of this region as an anchor since it defined both sequence and structure constraints. The alignment was then edited so as to maximize the number of base pairs in the consensus structure.

\section{Results}

\section{Northern Blot Verification of 7SK Sequences}

Numerous attempts to identify 7SK RNA in invertebrate phyla have been unsuccessful in the past. Neither RT-PCR experiments with mammalian primers nor northern blot analyses with oligonucleotide-primed cDNA probes were successful (Gürsoy et al. 2000). Therefore, we decided to increase northern blot sensitivity by using radioactively labeled antisense RNA probes. For this, the two most conserved (in comparison with the human sequence) regions of the lamprey (Lampetra fluviatilis) 7SK RNA gene (Gürsoy et al. 2000) were subcloned in inverted orientation under control of the T7 RNA polymerase promoter. Thus, these two clones allowed the synthesis in vitro of labeled transcripts with very high specific activity. The resulting two antisense RNA probes were complementary to regions 1-94 (A) and 283-316 (B) of lamprey 7SK RNA, respectively.

A northern blot obtained with cellular RNA of six vertebrate and six invertebrate organisms was consecutively hybridized with the aforementioned probes (Fig. 3). As is evident from the left side of Fig. 3, all vertebrate phyla

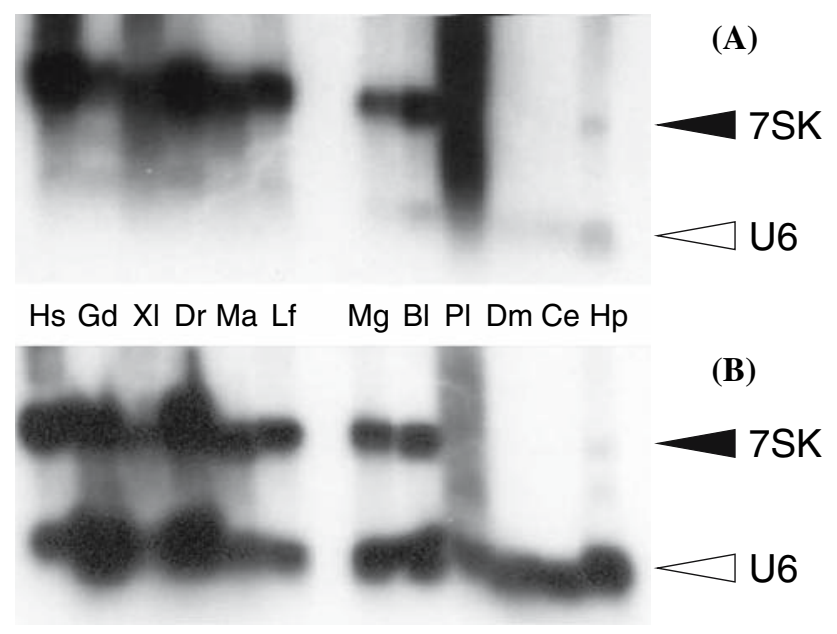

Fig. 3 Northern blot of cellular RNA from 12 different organisms using (A) $3^{\prime}$ - and (B) 5'-terminal 7SK antisense RNA for hybridization. Hs, Homo sapiens; Gd, Gallus domesticus; Xl, Xenopus laevis; Dr, Danio rerio; Ma, Mustelus asterias; Lf, Lampetra fluviatilis; $\mathrm{Mg}$, Myxine glutinosa; B1, Branchiostoma lanceolatum; Pl, Paracentrotus lividus; Dm, Drosophila melanogaster; Ce, Caenorhabditis elegans; Hp, Helix pomatia analyzed (human to lamprey) showed a clear hybridization signal with both the $3^{\prime}$-terminal (upper panel) and the $5^{\prime}$ terminal (lower panel) 7SK antisense RNA. In contrast, only hagfish (Myxine glutinosa) and two of the five invertebrates on the right side in Fig. 3 were found to be positive with respect to the 7SK antisense probes: amphioxus (Branchiostoma lanceolatum) and snail (Helix pomatia). Even longer exposure did not give a hint to any 7SK hybridization signal of either of the two probes with sea urchin (Paracentrotus lividus), fruitfly (Drosophila melanogaster), or nematode (Caenorhabditis elegans) RNA.

For normalization among samples, a labeled full-length antisense U6 snRNA has been included in both hybridizations. In the upper panel, weaker U6 signals are observed throughout. This is due to the rehybridization of the stripped blot. In both rounds, U6 antisense RNA hybridizes to the same target sequences, whereas the two 7SK probes bind to different areas of the 7SK RNA. The broad smear observed with sea urchin RNA is due to cross contamination of the 7SK antisense probe with ribosomal RNA and degradation products thereof.

\section{cDNA Cloning of Novel 7SK RNAs}

Cloning of invertebrate 7SK cDNA was performed by RTPCR and primers deduced from the most conserved elements of vertebrate 7SK RNA. Routinely, about 10 different primer combinations had to be tested. In many cases, PCR fragments with the expected lengths were obtained. After subcloning and sequencing, however, most fragments were found to represent pieces of ribosomal DNA. Only a single previously unidentified sequence was amplified from hagfish RNA. Two identical sequences, but differing in length, were obtained from amphioxus. A single new clone was obtained from snail. These clones showed a limited but significant sequence homology with the corresponding central sections of the vertebrate 7SK DNA. Therefore, the $3^{\prime}$ - and $5^{\prime}$-flanking sequences of those clones were amplified by RACE experiments. After subcloning and sequencing, composite 7SK RNA sequences were obtained for Myxine glutinosa (329 nt), Branchiostoma lanceolatum (304 nt), and Helix pomatia (303 nt).

These three 7SK RNA clones revealed sequence homology with lamprey 7SK DNA in the range between 44\% (snail) and 59\% (hagfish). The identification of several interspersed elements (7 to $11 \mathrm{nt}$ long) with a perfect match to the vertebrate 7SK RNA sequence strongly supported the notion that 7SK cDNA has been successfully cloned from two basal chordates (hagfish and amphioxus) and a single nondeteuterostome invertebrate. In addition, we report here sequences for Gadus morrhua and Mustelus asterias. All sequences have been deposited in GenBank, 
accession numbers AM773429-AM773436. Multiple sequence alignments can be found in the electronic supplement.

\section{Homology Search}

\section{Vertebrates}

Within vertebrates, homology search turned out to be rather straightforward. Simple blastn searches were sufficient. In eutherians, however, it is a problem to identify the functional 7SK gene among a larger number 7SK-derived pseudogenes. In fact, searching Ensembl v.44 with an $E$ value cutoff of $10^{-4}$ returns more than 100 hits in all eutherian genomes. In contrast, there are only 31 hits in Monodelphis domestica and 11 hits in the chicken genome. The current assembly of the genome of Xenopus tropicalis features two adjacent copies. These are identical also in an extended flanking sequence, indicating a recent segmental duplication of the locus or an assembly artifact; see, e.g., Cheung et al. (2003). In each of the five sequenced teleost fish, only a single copy of the 7SK is present. Three blast hits were found in the pre-Ensembl release of the sea lamprey genome. Only a single one, located on Contig17254, matches the published sequence from Lampetra fluviatilis over its full length.

In three vertebrate species, however, we failed to find a complete 7SK gene. Only a single partial hit was recovered from the low-coverage genome elephant shark Callorhynchus milli. All convincing blastn hits of the chicken 7SK sequence against the available Taeniopygia guttata shotgun reads seem to belong to a single locus. The corresponding sequence very well matches the chicken sequence but shows a 398-nt insert, which we interpret as an artifact. To our surprise, only a single blastn hit was found in the platypus genome. The corresponding sequence significantly deviates from the vertebrate consensus both in the first $\sim 8 \mathrm{nt}$ and in the last $\sim 100 \mathrm{nt}$, and it is not located in a region that is syntenic to the functional 7SK genes in other vertebrates. This blast hit thus is most likely a pseudogene. Since the locus around the platypus ICK homologue is incompletely assembled, it is reasonable to assume that we fail to find the platypus 7SK due to missing data, not because platypus has lost its functional 7SK RNA.

The functional 7SK genes can be recognized by the characteristic PSE sequence in their promotor regions; see Fig. 4. Furthermore, their genomic location between the glutathione S-transferase $\alpha$ GTA4 on the $5^{\prime}$ side and serine/ threonine protein kinase ICK on the $3^{\prime}$ side is conserved throughout most vertebrates. In Dasypus novemcinctus, the $3^{\prime}$ neighbor of $7 \mathrm{SK}$ is a homologue to "F-box only protein 9', (Fbox9). The same gene is found adjacent to the 7SK candidate in Sorex araneus. This is interesting because in most mammalia where the information is available (with the exception of Felis cattus and Tupaia belangeri, in which the 7SK snRNA lies between two glutathione transferase genes, possible an assembly artifact), Fbox 9 is usually the first gene $3^{\prime}$ on the same strand as 7SK. This is also observed in Gallus gallus and Xenopus tropicalis; in the latter no GTA4 homologue is annotated. Fbox9 is also located in close vicinity to the 7SK snRNA gene in teleosts except zebrafish.

\section{Basal deuterostomes}

While the vertebrate 7SK RNAs are very well conserved at sequence level (Gürsoy et al. 2000), blast searches soon reached their limits outside of this clade. A weak blast hit of

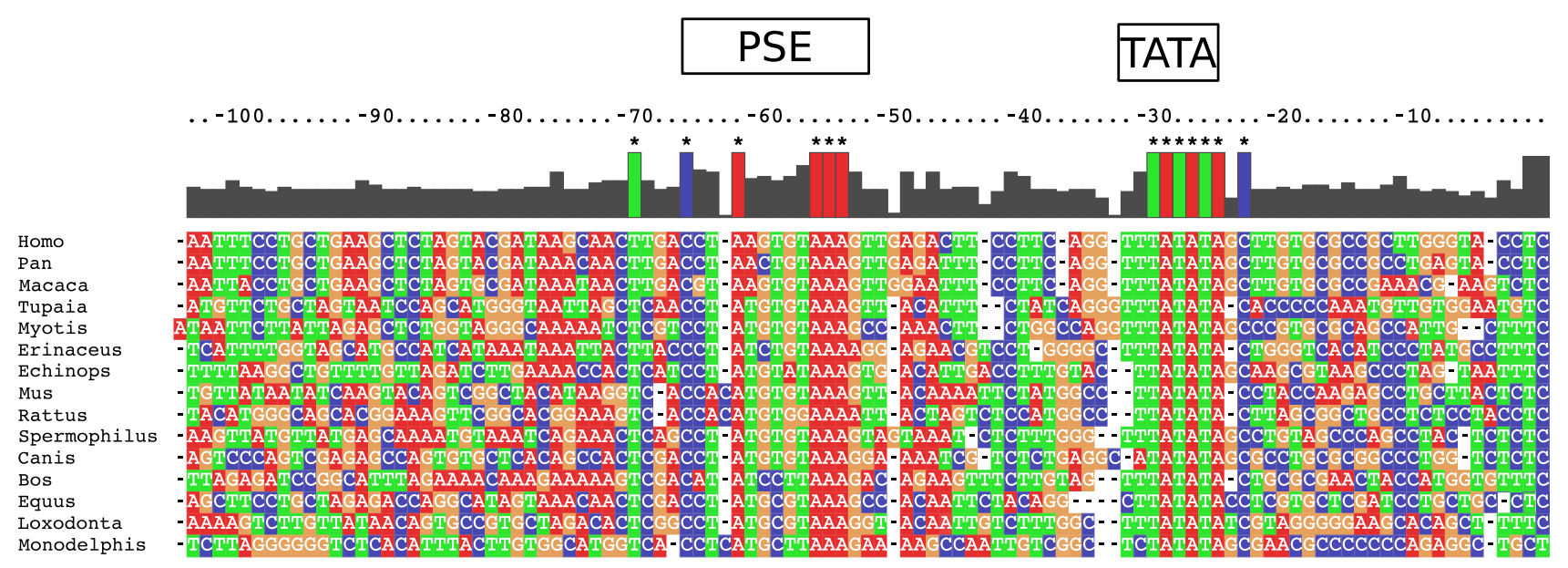

Fig. 4 Conservation of the the 7SK promoter region of the 7SK candidates located between the GTA4 and the ICK genes. In all mammals, both the "proximal sequence element" (PSE) and the TATA-box are easily recognizable 
the human query sequence in the Branchiostoma floridae genome was easily verified by comparison with the experimentally determined Branchiostoma lanceolatum 7SK RNA sequence. In total, we find six nearly identical 7SK candidates on five different scaffolds. These sequences are also nearly identical at least $100 \mathrm{nt}$ upstream of the 7SK. It is unclear whether there are really multiple functional copies of 7SK RNA dispersed in the amphioxus genome.

Beyond amphioxus, three further candidates were found by means of fragrep only: two closely related sequences from the urochordates Ciona intestinalis and Ciona savignyi, and a single candidate from the hemichordate Saccoglossus kowalevskii. In the $C$. intestinalis genome there is only a single 7SK locus. In contrast, the current assembly of $C$. savignyi features four nearly identical copies within $8 \mathrm{~kb}$ on reftig_107.

The PSE of basal deuterostomes, 63-48 nt upstream of $7 \mathrm{SK}$, is well conserved with the one of U6 snRNA for all investigated organisms. The TATA-box is located 32-25 nt upstream. An exception is Ciona intestinalis, which has an insertion of $15 \mathrm{nt}$ between the TATA-box and 7SK, and a 9-nt deletion between the PSE and TATA. The Ciona species shows no TATA-box conservation with other snRNAs, nevertheless, there is a slightly modified TATAbox. Branchiostoma has a canonical TATA-box.

Despite significant efforts we did not find credible candidates in the genome of the sea urchin Strongylocentrotus purpuratus. The three best candidates in this case lack the $3^{\prime}$ hairpin structure. In addition, the $5^{\prime}$ hairpin region can hardly be aligned with other deuterostome 7SK sequences. Our search also failed for the shotgun traces of the urochordate Oikopleura dioica. In this case we found a good candidate for the $3^{\prime}$ stem-loop structure, but the 300 nt upstream of this hit does not match other 7SK sequences. We suspect that these negative results might be due to incomplete genomic data in these cases.

\section{Protostome 7SK RNAs}

The fragrep search was successful in three protostome genomes: the mollusk Lottia gigantea and the two annelids Capitella capitella and Helobdella robusta. All three sequences are easily recognizable as homologues of the 7SK sequence that was cloned from the escargot Helix pomatia. In addition, a partial sequence from Aplysia californica was found by blast using the experimentally determined escargot 7SK sequence as query.

The PSE of Aplysia californica is located 67-41 nt upstream of 7SK snRNA; the sequence motif TGTATAGA matches the typical TATA-box sequence 35-28 nt upstream. In Lottia gigantea we find CTTATATA (positions -31 to -24 ) and the PSE $15 \mathrm{nt}$ upstream of the
TATA-box. In Capitella we find TATACA at positions -27 to -21 and a possible PSE, although it does not match well with the upstream sequence of the U6 snRNA in this species. The single shotgun read from Helobdella robusta does not show a recognizable TATA-box region but an alignable PSE region. It is not clear whether this sequence is a functional gene or a 7SK-derived pseudogene.

Despite extensive efforts, on the other hand, no 7SK candidate was found in any of the many available insect and nematode genomes. A search in the genomes of the two platyhelminths Schmidtea mediterranea and Schistosoma mansoni also remained unsuccessful. Thus, among protostomes, the 7SK RNA can be found only among lophotrochozoans.

Given the lack of success on ecdysozoan genomes and the four highly derived lophotrochozoan sequences, we were not surprised that searches in the genomes of diploblastic animals and in the choanoflagellate Monosiga brevicollis were also not successful.

\section{Refined Structural Models of 7SK RNAs}

The conserved structural features of the 7SK sequences are summarized in Fig. 5. Three structural elements are common to all known 7SK RNAs: the 5'stem, the $3^{\prime}$ stem, and a short hairpin structure (stem A) that should probably be considered as part of the $5^{\prime}$ structure. Vertebrate 7SK RNAs, in addition, share stem $\mathrm{B}$.

The secondary structures for the $5^{\prime}$ and $3^{\prime}$ stems were proposed already in previous publications. Wassarman and Steitz (1991) derived a model for the human 7SK snRNA based on chemical probing data. Egloff et al. (2006) used site-directed mutagenesis to demonstrate that both the $5^{\prime}$ and $3^{\prime}$ stems are functionally relevant. The structural model in Fig. 5 is derived from a sequence alignment that takes into account both sequence covariation and thermodynamic considerations. Our consensus model is in agreement with the previously published structures with a marginal exception: in Egloff et al. (2006) the regions marked in Fig. 5 are shown as an interior loop, while Wassarman and Steitz (1991) shows only the terminal A-U as part of the interior loop.

The $5^{\prime}$ stem models both for basal deuterostoma and for the lophotrochozoa are different in size, sequence, and structure. The only common ground among all three models is the GATC-GATC structure/sequence pattern at the beginning of the topmost stem.

The vertebrate-specific stem $\mathrm{B}$, which is not necessary for P-TEFb binding (Egloff et al. 2006), also fits very well with both experimental models, again, with a small difference affecting a small interior loop. It does not appear to have a counterpart in basal deuterostomes and protostomes. 


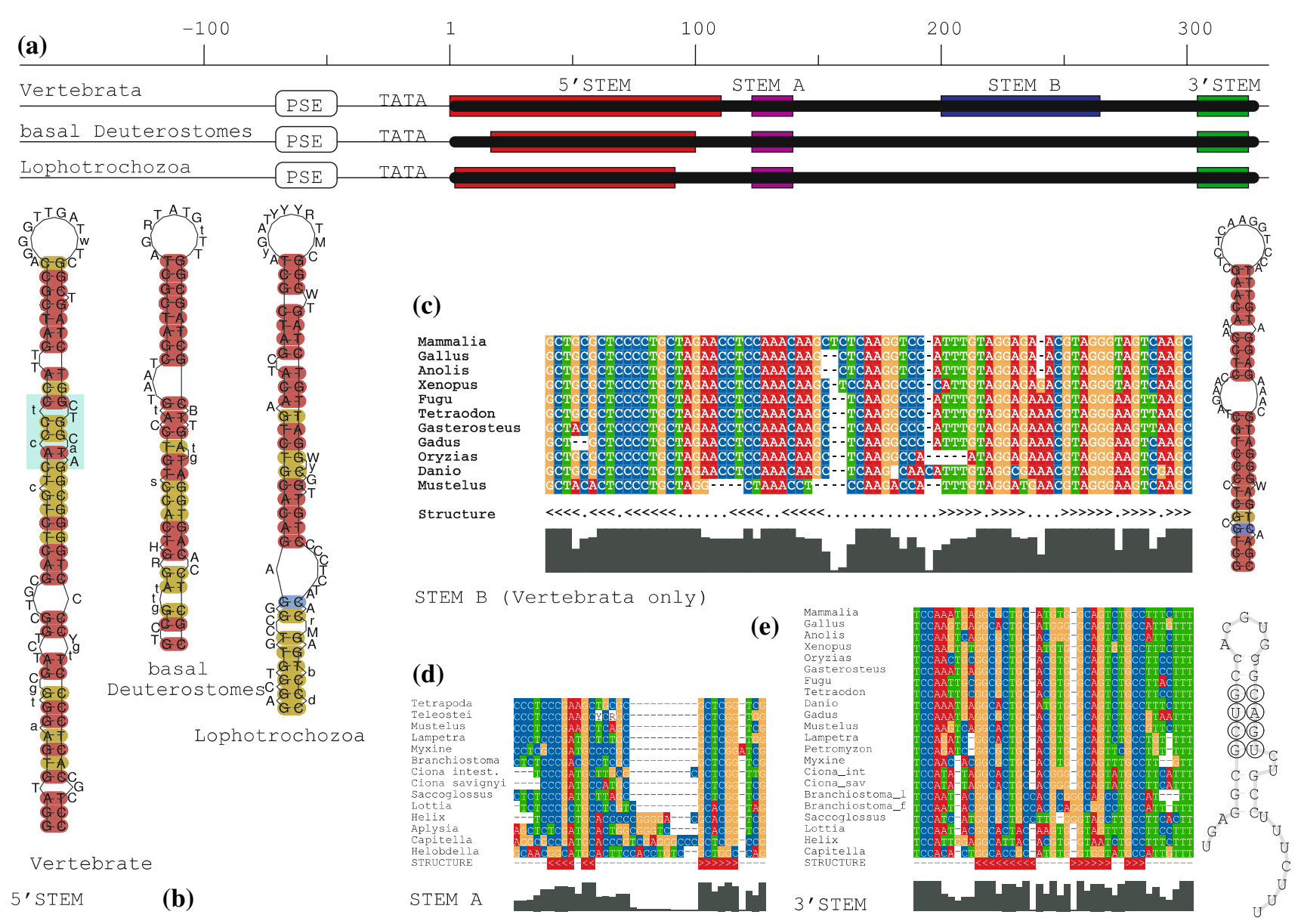

Fig. 5 Common structural elements of 7SK snRNAs. A The top panel schematically compares the location of upstream elements and RNA secondary features. While the structure of the $3^{\prime}$ stem is common to all 7SK snRNAs (except for the elongation of the stem by a GC pair in Branchiostoma and Saccoglossus), there are substantial cladespecific variations in the $5^{\prime}$ stem. The $7 \mathrm{SK}$ sequences of vertebrates and invertebrates do not differ significantly in length. Interestingly, vertebrate stem $\mathrm{B}$ corresponds to a region with no discernible structure in invertebrates. B Model for the terminal $5^{\prime}$ structure. With the exeption of marginal differences in the small region marked in the vertebrate $5^{\prime}$ stem, our consensus model is in complete agreement with previously published structures of vertebrate 7SK snRNAs (Egloff et al 2006; Wassarman and Steitz 1991). Conserved nucleotides in stems are shown in red; ocher (and circles in the $3^{\prime}$ stem, respectively) indicates consistent and compensatory mutations. C A common structure, stem B, in the "middle region," can be found only in vertebrates. D Structural alignment for stem A that can be found in all hits located next to the $5^{\prime}$-terminal structure. E Structural alignment and secondary structure drawing of the $3^{\prime}$-terminal hairpin
The central region of the $3^{\prime}$ stem is structurally conserved in all 7SK RNAs, the only exception being an extension of the most central stem by a single GC pair in Branchiostoma and Saccoglossus. The $3^{\prime}$ stem-loop structure can be extended by an additional 5 base pairs (bp) in vertebrates and, to a lesser extent, also in the other 7SK snRNAs. The exact pairing pattern in this extended region does not seem to be very well conserved, however.

The small stem A feature, finally, is highly conserved also in sequence across all known 7SK snRNA, although the size of the loop region is variable in the lophotrochozoan sequences. So far, no specific function has been reported for this region.

\section{Discussion}

Using both computational and "wet-lab" approaches we have shown that 7SK is phylogenetically much older than previously thought. While previously examples were known only from vertebrates, we found 7SK RNAs in most major deuterostome clades (chephalochordata, urochordata, and hemichordata) and in two lophotrochozoan clades (mollusca and annelida) (Fig. 6). In contrast, extensive efforts to find 7SK homologues in yeast and plants by us and others have remained unsuccessful. In these clades, no orthologue of HEXIM has been reported. Thus, most likely, neither fungi nor plants have the 7SK system. 

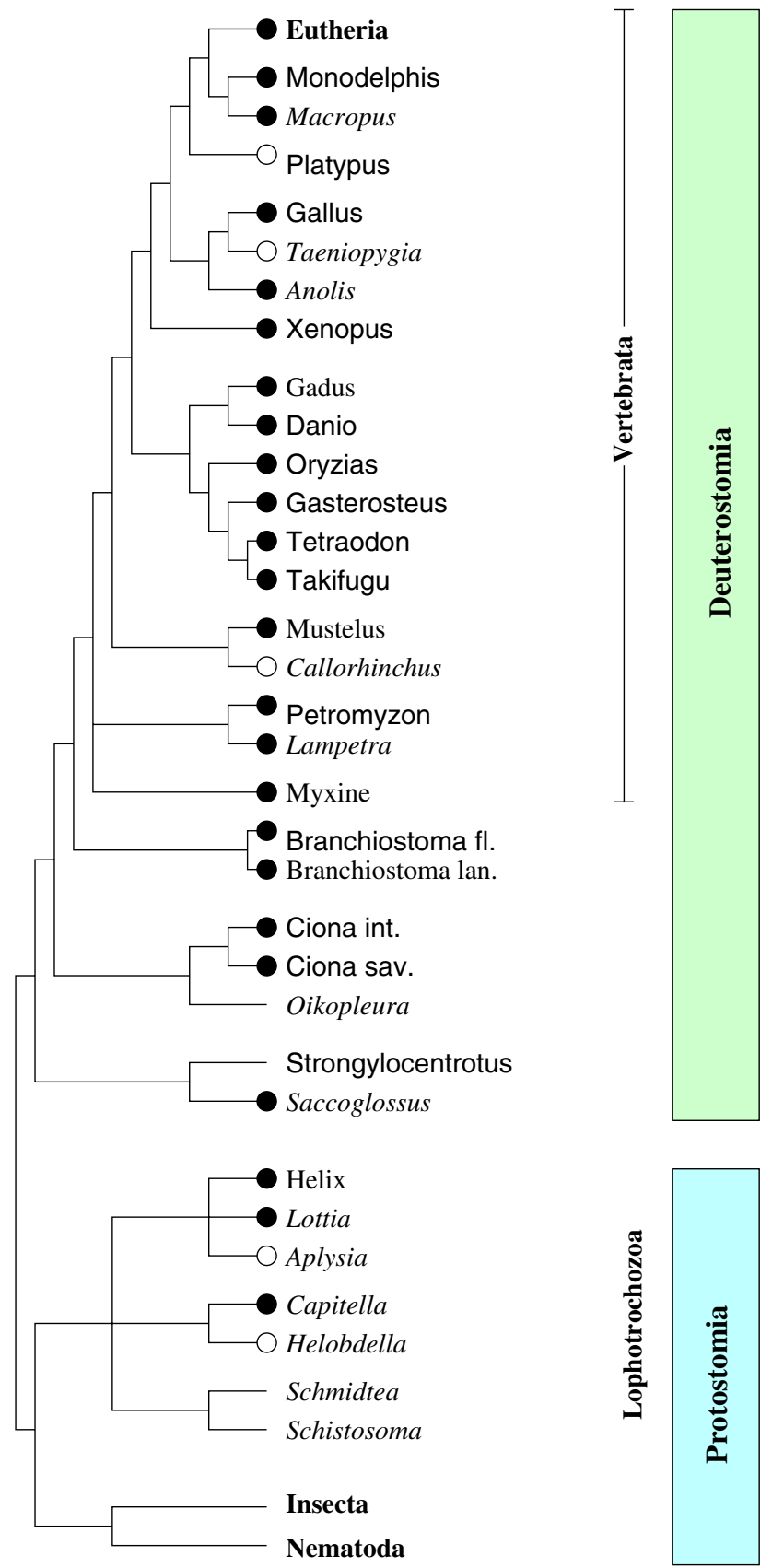

Fig. 6 Phylogenetic distribution of 7SK sequences. Boldface: clades in which multiple genomes were searched with consistent results. Sans serif font: assembled genomes. Italics: shotgun traces. Roman font: no genomic sequence available but positive experimental results. Filled circles indicate that a complete bona fide 7SK sequence was found; open circles mark species where we found a partial 7SK sequence. The phylogenetic tree follows a recent review (Telford 2006)]

Assuming that the commonly accepted sister-group relationship of Protostomia and Deuterostomia is indeed correct, our findings imply that the 7SK originated at latest in the bilaterian ancestor. In contrast, we found no trace of a 7SK RNA candidate either in platyhelminthes, in any of the numerous ecdysozoan species for which genomic data are available, or in the genome of the cnidarian Nematostella vectensis. In summary, our data support an (early) bilaterian origin for 7SK snRNA.

The monophyly of the Ecdysozoa is, among other arguments, also supported (Telford 2006) by the shared secondary absence of large numbers of genes in euarthropods and nematodes (Hughes and Friedman 2004). There is no functionally described HEXIM1 orthologue in insects. The current release of the ENSEMBL (v. 44) homology annotation, however, lists HEXIM homologues in Drosophila melanogaster (CG3508), Aedes aegyptii (AAEL013291), and Anopheles gambiae (AGAP002875). It is at least conceivable, therefore, that an ancestral 7SK gene has been secondarily lost in this clade. Alternatively, the 7SK sequence might have diverged so far that it is not recognizable with currently available bioinformatic approaches.

The analysis of sequences and secondary structures revealed a striking difference between vertebrate and invertebrate sequences. While vertebrate 7SK RNAs are highly conserved in both sequence and structure, the molecule is highly variable in the other clades. Consensus structure models derived using a combination of thermodynamic folding and evaluation of compensatory mutations reveal three structural motifs that are conserved throughout all known 7SK sequences. The central domain (stem B), however, is present in vertebrates only, while elsewhere this region is so variable that our attempts to construct plausible alignments failed.

\section{Supporting Material}

Supplemental data, in particular, machine-readable sequence alignments, tables of genomic coordinates, and lists of PSE elements can be found at http://www.bioinf. uni-leipzig.de/Publications/SUPPLEMENTS/07-021/.

Acknowledgments This work has multiple roots: Dorota KoperEmde's Ph.D. thesis at the Ruhr University Bochum (2004), Manja Marz' M.Sc. thesis at the University of Leipzig (2006), and an Advanced Bioinformatics Computer Lab Course on "RNA Homology Search" organized by PFS at the University of Vienna in Fall 2006. It was supported in part by the DFG Bioinformatics Initiative and the GK Wissensrepräsentation (Leipzig) and by the Austrian GEN-AU projects "Non-Coding RNA" and "Bioinformatics Integration Network II" (Vienna). We thank Guido Fritzsch for taking care of the Genbank submissions.

\section{References}

Bailey T, Elkan C (1994) Fitting a mixture model by expectation maximization to discover motifs in biopolymers. In: Proceedings of the Second International Conference on Intelligent Systems for Molecular Biology. AAAI Press, Menlo Park, CA, pp 28-36 
Blazek D, Barboric M, Kohoutek J, Oven I, Peterlin BM (2005) Oligomerization of HEXIM1 via 7SK snRNA and coiled-coil region directs the inhibition of $\mathrm{P}-\mathrm{TEFb}$. Nucleic Acids Res 33:7000-7010

Cheung J, Estivill X, Khaja R, MacDonald JR, Lau K, Tsui LC, Scherer SW (2003) Genome-wide detection of segmental duplications and potential assembly errors in the human genome sequence. Genome Biol 4:R25 (2003)

Chomczynski P, Sacchi N (1987) Single-step method of RNA isolation by acid guanidinium thiocyanate-phenol-chloroform extraction. Anal Biochem 162:156-159

Dahlberg JE, Lund E (1988) The genes and transcription of the major small nuclear RNAs. In: M. Birnstiel (ed) Structure and function of major and minor small nuclear ribonucleoprotein particles. Springer-Verlag, Berlin, pp 38-70

Egloff S, Van Herreweghe E, Kiss T (2006) Regulation of polymerase II transcription by 7SK snRNA: two distinct RNA elements direct P-TEFb and HEXIM1 binding. Mol Cell Biol 26:630-642

Frohman MA, Dush MK, Martin GR (1988) Rapid production of fulllength cDNAs from rare transcripts: amplification using single gene-specific oligonucleotide primer. Proc Natl Acad Sci USA 85:8998-9002

Griffiths-Jones S (2005) RALEE—RNA ALignment editor in Emacs. Bioinformatics 21:257-259

Gürsoy HC, Koper D, Benecke BJ (2000) The vertebrate 7S K RNA separates hagfish (Myxine glutinosa) and lamprey (Lampetra fluviatilis). J Mol Evol 50:456-464

He WJ, Chen R, Yang Z, Zhou Q (2006) Regulation of two key nuclear enzymatic activities by the 7SK small nuclear RNA. Cold Spring Harb Symp Quant Biol 71:301-311

Hernandez G Jr, Valafar F, Stumph WE (2007) Insect small nuclear RNA gene promoters evolve rapidly yet retain conserved features involved in determining promoter activity and RNA polymerase specificity. Nucleic Acids Res 35:21-34

Hofacker IL, Fekete M, Stadler PF (2002) Secondary structure prediction for aligned RNA sequences. J Mol Biol 319:10591066

Hughes AL, Friedman R (2004) Shedding genomic ballast: extensive parallel loss of ancestral gene families in animals. J Mol Evol 59:827-833
Krüger W, Benecke BJ (1987) Structural and functional analysis of a human 7 S K RNA gene. J Mol Biol 195:31-41

Marz M, Kirsten T, Stadler PF (2007) Evolution of spliceosomal snRNA genes (manuscript in preparation)

Michels AA, Fraldi A, Li Q, Adamson TE, Bonnet F, Nguyen VT, Sedore SC, Price JP, Price DH, Lania L, Bensaude O (2004) Binding of the 7SK snRNA turns the HEXIM1 protein into a PTEFb (CDK9/cyclin T) inhibitor. EMBO J 23:2608-2619

Moon IS, Krause MO (1991) Common RNA polymerase I, II, and III upstream elements in mouse 7SK gene locus revealed by the inverse polymerase chain reaction DNA. Cell Biol 10:23-32

Mosig A, Chen JL, Stadler PF (2007) Homology search with fragmented nucleic acid sequence patterns. In: Giancarlo R, Hannenhalli S (eds) WABI 2007. Lecture Notes in Computer Science, Vol 4645. Springer Verlag, Berlin, pp 335-345

Murphy S, Altruda F, Ullu E, Tripodi M, Silengo L, Melli M (1984) DNA sequences complementary to human 7 SK RNA show structural similarities to the short mobile elements of the mammalian genome. J Mol Biol 177:575-590

Murphy NS, Di Liegro C, Melli M (1987) The in vitro transcription of the 7SK RNA gene by RNA polymerase III is dependent only on the presence of an upstream promoter. Cell 51:81-87

Myslinksi E, Krol A, Carbon P (2004) Characterization of snRNA and snRNA-type genes in the pufferfish Fugu rubripes. Gene 330:149-158

Peterlin BM, Price DH (2006) Controlling the elongation phase of transcription with P-TEFb. Mol Cell 23:297-305

Reddy R, Henning D, Subrahmanyam CS, Busch H (1984) Primary and secondary structure of 7-3 (K) RNA of Novikoff hepatoma. J Biol Chem 259:12,265-12,270

Telford M (2006) Animal phylogeny. Curr Biol 16:R981-R985

Wassarman DA, Steitz JA (1991) Structural analyses of the 7SK ribonucleoprotein (RNP), the most abundant human small RNP of unknown function. Mol Cell Biol 11:3432-3445

Zieve G, Penman S (1976) Small RNA species of the HeLa cell: metabolism and subcellular localization. Cell 8:19-31 